\title{
Soluble insulin receptor and metabolic syndrome
}

Marcia Hiriart-Urdanivia, * Carmen Sánchez-Soto, Myrian Velasco, Jean Sabido-Barrera, and Rosa Isêja Ortiz-Huidobro

Universidad Nacional Autónoma de México, Institute of Cell Physiology, Department of Cognitive Neuroscience, Mexico City, Mexico

\begin{abstract}
The metabolic syndrome describes a group of signs that increase the likelihood for developing type 2 diabetes mellitus; cardiovascular diseases and some types of cancer. The action of insulin depends on its binding to membrane receptors on its target cells. We wonder if blood insulin could travel bound to proteins and if, in the presence of hyperinsulinemia, a soluble insulin receptor might be generated. We used young adult Wistar rats (which have no predisposition to obesity or diabetes), whose drinking water was added $20 \%$ of sugar and that were fed a standard diet ad libitum for two and six months. They were compared with control rats under the same conditions, but that had running water for consumption. At two months, the rats developed central obesity, moderate hypertension, high triglyceride levels, hyperinsulinemia, glucose intolerance anid insulin resistance, i.e., metabolic syndrome. Electrophoresis of the rats' plasma proteins was performed, followed by Western Blot (WB) for insulin and for the outer portion of the insulin receptor. The bands corresponding to insulin and to the receptor external part were at the same molecular weight level, 25-fold higher than that of free insulin. We demonstrated that insullin, both in control animals and in those with hyperinsulinemia, travels bound to the receptor outer portion (ectodomain), which we called soluble insulin receptor, and that is released al higher amounts in response to plasma insulin increase; in rats with metabolic syndrome and hyperinsulinemia, plasma levels are much higher than in controls. Soluble insulin receptor increase in blood might be an early sign of metabolic syndrome.
\end{abstract}

KEY WORDS: Soluble insulin receptor. Hyperinsulinemia. Insulin resistance.

\section{The health problem}

The $21^{\text {st }}$ century started out with an overweight and obesity epidemic. The number of people with obesity in the world has doubled from 1980 to date. Approximately $40 \%$ of the world adult population (1900 million) has overweight and $13 \%$, obesity. In Mexico, data are not better: according to the 2016 National Health and Nutrition Survey, seven out of every 10 adults are overweight or obese. To increase the alarm, three out of every 10 school-age children and four out of every 10 adolescents are also overweight, with the problems this entails. It is necessary to understand how the signs of the metabolic syndrome develop and progress towards type 2 diabetes mellitus (DM2).
Even when there are numerous articles on metabolic syndrome and DM2 in the literature that focus on clinical manifestations, there are basic concepts of insullin physiology that are not understood.

\section{Metabolic syndrome and DM2}

Metabolic syndrome is a set of signs that increases the likelihood of developing DM2, cardiovascular diseases and some types of cancer. Metabolic syndrome is regarded as the manifestation of three of the ofollowing signs: central obesity, hypertension, dyslipidemia, hyperinsulinemia, impaired fasting glucose and insulin resistance. Counteracting them can prevent the development of more severe pathologies, such as DM2. ${ }^{1,2}$
Correspondence:

${ }^{*}$ Marcia Hiriart-Urdanivia

E-mail: mhiriart@ifc.unam.mx
Date of reception: 25-03-2018

Date of acceptance: 27-03-2019

DOI: 10.24875/GMM.M20000339
Gac Med Mex. 2019;155:5000503

Contents available at PubMed

www.gacetamedicademexico.com

0016-3813/@ 2019 Academia Nacional de Medicina de México, A.C.. Published by Permanyer. This is an open access article under the CC BY-NG-ND license (http://creativecommons.org/licenses/by-nc-nd/4.0/). 
DM2 is a disease that manifests itself with hyperglycemia, secondary to a depletion of beta cells, the only cells in the body that produce and secrete insulin. Beta cells are referred to as being depleted when they cannot maintain glucose homeostasis (fasting glucose $<125 \mathrm{mg} / \mathrm{dL}$ ); it is a multifactorial, polygenic disease that depends on internal factors such as microbiota and external factors such as diet, exposure to environmental pollutants, etc. In Mexico, the genetics of the mestizo population indicates that it is more prone to develop DM2.

Often, before hyperglycemia occurs, there is insulin resistance (there is insulin production but the body does not respond to it), in addition to beta cell excessive stimulation, which drives them to exhaustion. This explains that the metabolic syndrome precedes DM2.

During development, puberty and pregnancy there are temporary stages of physiological insulin resistance, which are associated with periods of hyperinsulinemia and hyperglycemia.

\section{Physiology of insulin and its receptor}

Insulin is a peptide hormone, exclusively secreted by pancreatic beta cells as a zinc-stabilized hexamer, which dissociates and transports the active form of insulin, which is formed by subunits $A$ and $B$, between which there are three bridges, two disulfide and one intracatenary bridge in subunit $A$.

The most important stimulus in insulin secretion is alpha d-glucose, but some amino acids and fatty acids are also involved. When eating, nutrients are absorbed and reach the pancreas through the portal system. When glucose concentration increases, insulin secretion increases. Beta cell converts chemical into electrical signals, and insulin granules are secreted when calcium intracellular concentration is increased. ${ }^{3}$ It is generally accepted that the hormone travels free in the blood.

All body cells have insulin receptors, but they are more abundant and their action is clearer in the liver, fat and skeletal muscle. Insulin receptors belong to the family of receptors with tyrosine kinase activity and are heterodimers formed by two subunits, alpha and beta. The alpha subunit binds insulin and beta has the kinase domain. The alpha and beta subunits are connected by a disulfide bridge and the alpha subunits have two to four bridges. It has been observed that each subunit can bind one insulin molecule with high affinity and another with low affinity. ${ }^{4}$

Insulin binding to the receptor initiates the signal and continues with a conformational change that produces
Hiriart-Urdanivia M, et al.: Insulin soluble receptor

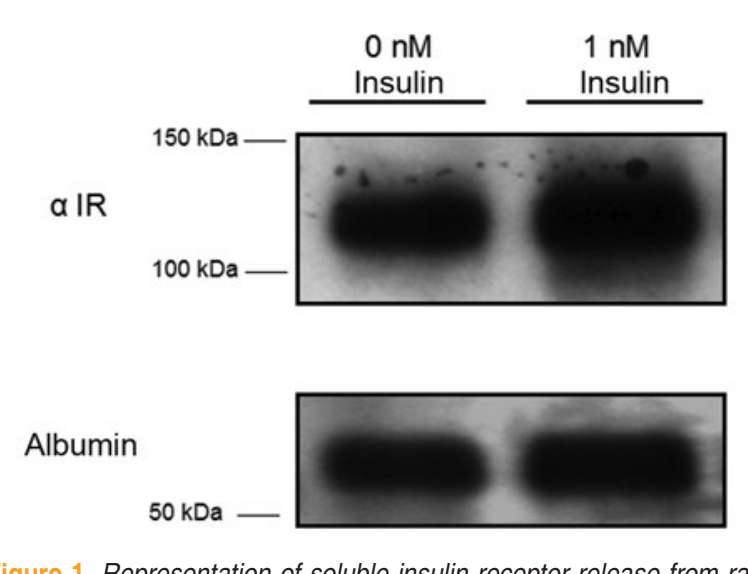

Figure 1. Representation of soluble insulin receptor release from rat adipocytes. Immunoblot for the insulin receptor alpha chain in the culture medium of isolated adipocytes. The cells $\left(2 \times 10^{6}\right.$ cells) were treated with $1 \mathrm{nM}$ insulin for 30 minutes, then the cell culture mediumwas obtained, which was concentrated by filtration for later analysis. The control group was stimulated with $0.001 \%$ dimethylsulfoxide and as load control, immunoblot for albumin was performed.

transphosphorylation of the beta subunits, which initiates a protein-to-protein chain of intracellular phosphorylation, which leads to Akt2 promoting the transport of Glut4 to the membrane and an increase in glucose entry, and glycogen, triglyceride and protein synthesis, depending on the tissue involved, and also the activation of mitogen-activated protein kinase, which mediates gene expression regulation. ${ }^{4}$

\section{Development of a metabolic syndrome animal model}

We chose Wistar rats because they have no tendency towards obesity or to develop diabetes: young adults of two and a half months; they were fed with a normal diet and water with $20 \%$ of sugar added. We compared them with their controls two and six months after treatment was started. Since the comparison at two months, the male rats were identified to have developed central obesity, moderate hypertension, elevated triglycerides, glucose intolerance and insulin resistance; i.e., metabolic syndrome. ${ }^{2,5}$ We observed that the development of metabolic syndrome markedly differed between genders: at six months, the male rats in the experimental group had a more pronounced metabolic syndrome; initially, females were more glucose intolerant and it took them longer to develop the syndrome.

We have observed that, in metabolic syndrome,the liver becomes fatty; fat itself, whose cells grow much, secrete many hormones and are invaded by white blood cells; the body feels swollen. Beta cells secrete a large amount of insulin, leading to hyperinsulinemia, a condition that, if prolonged, makes for cells to become 


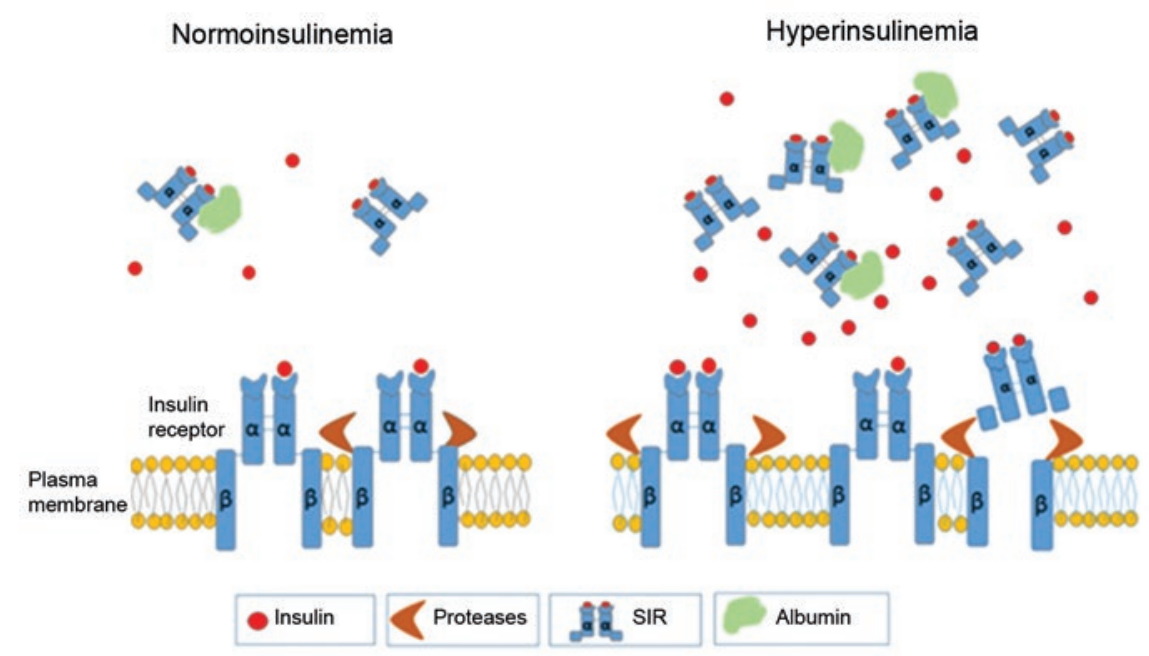

Figure 2. Insulin receptor in control hepatocytes and hepatocytes treated with insulin $1 \mathrm{nM}$. In the control hepatocytes, insulin receptor was tified in the membrane; when insulin was increased, protease activity was increased, as it occurs with hyperinsulinemia, where there is protease activity and more soluble insulin receptor ectodomain is shed.

depleted and for type 2 diabetes to develop. At six months of having started the experiment, the rats were more obese and beta cells began to decay and not secrete enough insulin to maintain blood glucose at normal levels, especially in males.

\section{The transport of insulin in plasma}

Since it is a peptide, insulin is thought to travel free in the blood to its target organs. However, it has recently been shown that only a small fraction of insulin travels free in blood; the rest of the hormone travels bound the insulin receptor ectodomain (soluble insulin receptor).

We analyzed, in vitro, the release of the receptor alpha subunit in rat hepatocytes, since they are rich in insulin receptor. When increasing concentrations of insulin were applied, it was clear that the release increases significantly when the concentration of insulin in the extracellular medium increases, reaching a maximum of $1 \mathrm{nM}$ of insulin. This release is prevented if the hepatocyte incubation medium includes a protease inhibitor. ${ }^{3}$ Insulin can bind to albumin but with lower affinity. We do not know if protein-to-protein complexes dissociate, allowing insulin to interact with membrane insulin receptors.

\section{Mechanisms of resistance to hyperinsulinemia}

Hyperinsulinemia is incompatible with life, and thus mammals developed mechanisms to counteract it. In the short term, the soluble insulin receptor can neutralize excess secreted insulin; in the long term, tissues can become resistant to the hormone, in addition to continue neutralizing it via the soluble insulin receptor.

The amount of receptor was higher in rats with metabolic syndrome than in control rats, which is probably related to hyperinsulinemia. Incubation of hepatocytes with insulin and a protease inhibitor decreases the amount of soluble insulin receptor released into the medium. Recently, we have verified the presence of this same phenomenon in rat adipocytes by incuibating them with insulin at a concentration of $1 \mathrm{nM}$ : there was an increase in the release of soluble receptor in comparison with control rats (Figure 1).

\section{Soluble insulin receptor in diabetes and metabolic syndrome}

A group of researchers showed that, in patients with diabetes, a soluble form of insulin receptor could be detected, ${ }^{6}$ which they identified as a health problem in response to hyperglycemia rather than a physiological condition. Part of that group continued working on the subject and described that this event could be part of insulin resistance. They demonstrated a positive correlation between soluble insulin receptor and $\frac{\digamma}{g} \mid u-$ cose extracellular concentration and glycosylated themoglobin (A1C). ${ }^{7}$

Not only has the insulin receptor ectodomain been found in plasma, but also in leptin, tumor necrosis factor alpha, etc. This phenomenon is known as shedding (membrane shedding) and is carried out by proteases. In the case of the soluble insulin receptor in Hep2 itver tumor cells, the authors suggest that it is not an MMP metalloprotease, or an ADAM-type enzyme, because 
they are not detected in their assays. The authors propose that the higher the receptor glycosylation, the easier it is for it to be attacked by proteases. In addition, this model requires modified $\mathrm{O}$ - $\mathrm{N}$-acetyl-linked glucosamine and a calcium-dependent protease for the shedding of the receptor to occur. ${ }^{7}$

Our group has found that rats with metabolic syndrome have much higher soluble receptor plasma levels, which can be correlated with the increase in insulin. Furthermore, we have found that soluble insulin receptor plasma levels are more increased in rats with higher degrees of hyperinsulinemia (Fig. 2). In females, insulin does not increase so much six months after having been ingesting the water with sugar and this is also correlated with a lower level of circulating soluble insulin receptor.

\section{Perspectives}

The results of circulating insulin binding with the soluble insulin receptor are of clinical interest both for diagnosis and for blood levels monitoring. On the other hand, treatment with a specific enzyme that inhibits receptor proteolysis could increase solublevinsulin receptor levels and the amount of free insulin in plasma, which is the biologically active hormone. 预e study of insulin binding to plasma proteins could Fead to finding some way to limit insulin resistance.

\section{References}

1. Hiriart M, Velasco M, Larqué $C$, Díaz-García CM. Metabolic synâfome and ionic channels in pancreatic beta cells. Vitam Horm. 2014;95:872114.

2. Velasco M, Larqué C, Gutiérrez-Reyes G, Arredondo R, Sánchez-Soto $\mathrm{C}$, Hiriart M. Metabolic syndrome induces changes in KATP-channels and calcium currents in pancreatic $\beta$-cells. Islets. 2012;4:302-311

3. Hiriart M, Sánchez-Soto C, Díaz-García CM, Castanares DT, Avitia M, Velasco M, et al. Hyperinsulinemia is associated with increased sojuble insulin receptors release from hepatocytes. Front Endocrinol (Lausanne). 2014:5:95.

4. Tatulian SA. Structural dynamics of insulin receptor and transmembrane signaling. Biochemistry. 2015;54:5523-5532.

5. Larque C, Velasco M, Navarro-Tableros V, Duhne M, Aguirre J, Gutiérrez-Reyes G, et al. Early endocrine and molecular changes in mètabolic syndrome models. IUBMB Life. 2011;63:831-839.

6. Soluble Insulin Receptor Study Group. Soluble insulin receptor ectodomain is elevated in the plasma of patients with diabetes. Diabetes. 2007;56:2028-2035.

7. Yuasa T, Amo K, Ishikura S, Nagaya H, Uchiyama K, Hashida St al. Development of in vitro model of insulin receptor cleavage induced by high glucose in HepG2 cells. Biochem Biophys Res Corhmun. 2014; $445: 236-243$ 